\title{
Management of Diarrhea - Changing Trends in Last 50 Years
}

\author{
AK Patwari \\ Department of Pediatrics, Hamdard Institute of Medical Sciences, Hamdard University, New Delhi, India \\ akpatwari@gmail.com
}

$\mathrm{I}$

n January 1968, the 50-paged issue of Indian Pediatrics included 4 original articles and 2 case reports. Amongst these, we decided to review the leading original article on "Trends in the Treatment of Acute Diarrhoeas in Infancy" by Udani, et al. [1] as diarrheal diseases continue to be a major cause of morbidity and mortality in under-five children. Last 50 years have witnessed a great deal of progress in understanding of etiology, pathogenesis and management of diarrhea, particularly after the discovery of scientific basis of glucose-linked sodium absorption in diarrhea. Even though the ultimate quest for an effective 'antidiarrheal drug' continues, through this communication, we present the advances made over the past 50 years in knowledge and practices of management of diarrhea in children.

\section{The Past}

The scenario described by Udani, et al. [1], half a century ago, highlights the magnitude of diarrheal diseases in children based on hospital-based data from Mumbai, Madras, Vellore and Delhi. As expected, 89\% children who reported to these hospitals were less than 2 years of age, and $93 \%$ of diarrheal deaths in children occurred in this vulnerable age group. Most of the research interest in those days was focused on etiology and sensitivity of isolated organisms to available spectrum of antibiotics. Different pathogenic organisms were reported in $66 \%$ of cases but Shigella group of organisms known to cause invasive diarrhea were isolated only in $8 \%$ of cases [1]. As serotyping of $E$. coli was not routinely available at that time, it is difficult to say what proportion of those $44 \%$ cases in whom E.coli was isolated were actually infected by diarrheagenic strains. Viruses isolated in $32 \%$ cases included Polio, Echo, Coxsackie and unidentified viruses. Since Rotavirus was discovered much later in
1973, one cannot comment on contribution by Rotavirus and other viruses known to cause diarrhea in children.

However, the available research data did influence the management protocol of diarrhea, which at that time heavily depended on use of antibiotics. Apart from antibiotics, the mainstay of treatment was intravenous fluid therapy in almost all the cases, which could have been responsible for a high proportion of cases with hyponatremia (33-62\%), hypernatremia (4-15\%) and hypokalemia (18-88\%). Despite the fact that $40 \%$ children in the study had poor nutritional status, a 24 hours starvation was recommended while the child received only parenteral fluid therapy. This hospital-based study indirectly reflects the common management practices of diarrheal diseases followed all across the country in those days.

Historical background and past knowledge: Description of diarrhea as a health problem dates back to Vedic era, and the condition is included in Bhaisajya Suktas of Atharva Veda. Sushruta, the father of Ayurveda, had prescribed that cholera victims are to be "given to drink a profuse quantity of tepid water in which rock salt and molasses have been dissolved; or clarified water combined with rice gruel" [Sushruta Samhita III, verse II], highlighting the concern for loss of water and electrolytes during an episode of diarrhea. However, with the advent of modern medicine and available knowledge about etiology and pathogenesis, the emphasis shifted more towards microorganisms and antibiotics. As described by Udani, et al. [1], during 1960's standard management of diarrhea in children revolved around intravenous fluid therapy and antibiotics like streptomegma, walamycin and chloramphenicol. The list of these so called 'antidiarrheal' antibiotics kept on changing to tetracyclines, cotrimoxazole, furozolidine etc. depending upon isolation of different 
microorganisms and their sensitivity patterns. Pectin and bismuth kaolin were two other pharmacological agents commonly used to 'reduce' water content of diarrheal stools. It was later proved beyond doubt that these binding agents simply changed the cosmetic appearance of stool without reducing volume of water lost in diarrheal stools [2]. It took several years to understand that all strains of E. coli do not cause diarrhea, and most of them are self-limiting and therefore do not need antibiotics.

Scientific basis of glucose-linked absorption of sodium was considered a major breakthrough in the management of diarrhea in 1960s, and Lancet considered it potentially the most important medical advance of the century. However, the concept of Oral Rehydration Salt (ORS) was mostly revered than practiced, and the major thrust remained on intravenous fluids, antibiotics, antiprotozoals and antiemetics. It was during 1971 IndoPak war when clinical efficacy of ORS was actually demonstrated in thousands of children and adults suffering from diarrhea in refugee camps in Dhaka and Calcutta. Following this large-scale experience, the clinical superiority of ORS in preventing and treating dehydration, and replacing intravenous therapy was accepted as a major public health intervention. World Health Organization promoted ORS as a universal solution for preventing and treating diarrheal dehydration due to any etiology and in all age groups. By 1980s, introduction of Oral Rehydration Therapy (ORT) had a motivating influence on diarrhea management practices in academic institutions. ORS was acknowledged as appropriate solution for prevention and treatment of dehydration but prescription of antibiotics continued because children with high fever were invariably prescribed antibiotics [3]. However, very little change took place in office practice of pediatricians. Besides, academicians and researchers continued with their own doubts on efficacy and safety of WHO-ORS $\left(\mathrm{Na}^{+} 90\right.$ $\mathrm{mEq} / \mathrm{L}$ and osmolality $331 \mathrm{mOsmol} / \mathrm{L}$ ) challenging its composition. Whereas some researchers had genuine concerns about safety of WHO-ORS in malnourished children and neonates because of risk of hypernatremia [4], some others argued against using WHO-ORS as a universal rehydrating solution because of its higher sodium content in comparison to sodium losses in noncholera stools [5]. Promotion of Salt Sugar Solution as an alternative to WHO-ORS to treat dehydration in situations when WHO-ORS packets were not available lead to serious consequences in some children when the solution was prepared incorrectly. This further limited use of ORS as the corner stone of diarrhea case management for many years.
Introduction of ORT did revolutionize public health approach to diarrheal diseases with a significant decline in diarrheal deaths over the years. However, the major concern for practicing physicians continued because of their quest for improved or super ORS which could reduce purge rate and stool volume. Some clinical trials showed promising results with ORS based on rice, lentils and alanine [6] but in actual practice, no significant clinical benefit was observed and therefore the promising entry of these brands of so called 'improved ORS' was short-lived.

Concept of early repair of gut mucosa with continued feeding [7] replaced the old practice of 'rest to gut' or 'no feeding in first 24 hours'. Continuation of breastfeeding and feeding during diarrhea became an essential component of ORT, particularly children with malnutrition are offered food right from the beginning of rehydration phase. In order to take care of nutritional consequences, one extra feed for 2 weeks is recommended after an episode of acute diarrhea to ensure adequate weight gain.

Some antisecretory and ant motility agents were actively marketed in India during 1970s and 1980s. Even though some of these drugs are safely used in Traveler's diarrhea in adult population, their routine use in pediatric practice was considered unsafe because of a narrow safety margin between the effective therapeutic dose and the toxic dose. After 'lopermide tragedy in Pakistan in 1980 ' when a number of children died because of toxic doses of loperamide, liquid formulations of the drug was banned in several countries.

Considering the magnitude of diarrheal diseases in children and the high under-five mortality because of diarrheal deaths, WHO launched Control of Diarrheal Diseases (CDD) Program in 1978, which was soon followed by Govt. of India as National Control of Diarrheal Diseases (NCDD) Program in 1980. Ministry of Health and Family Welfare established a separate 'ORT Section' to promote standard case management of diarrhea by establishing Diarrhea Training and Treatment Units (DTTUSs) in medical colleges, Mini DTUs in District Hospitals and ORT Corners in Primary Health Centers. In 1992, NCDD program was merged with Child Survival and Safe Motherhood (CSSM) program which was later converted to Reproductive and Child Health (RCH) Program.

\section{The Present}

Diarrheal diseases continue to be one of the commonest childhood illnesses in India even though the under-five mortality due to diarrheal deaths has significantly 
reduced. Current case management practices are based on WHO guidelines [8] that primarily focus on ORT (using low osmolarity ORS, continued feeding/increased breastfeeding), zinc supplementation and appropriate use of antibiotics (in dysentery, cholera, severe malnutrition and associated infections). In 2003, WHO and UNICEF recommended use of low osmolarity ORS $(245 \mathrm{mOsm} / \mathrm{L})$ based on multiple clinical trials showing that the reduced osmolarity solution reduced stool volume in children with diarrhea as well as the need for intravenous fluid therapy. Following a comprehensive review by WHO Task Force on Management of Acute Diarrhea, Indian Academy of Pediatrics (IAP) also recommended low osmolarity ORS for prevention and treatment of dehydration, and zinc supplementation (10$20 \mathrm{mg}$ of elemental zinc) for 14 days [9]. Introduction of Rotavirus vaccine in the immunization schedule in addition to increase in measles vaccine coverage are other potentially effective interventions to significantly decrease the incidence and severity of diarrhea in children.

The current management guidelines are effective in reducing mortality, and to some extent reduction in stool volume during an episode of acute diarrhea. However, ORS does not markedly reduce volume and frequency of loose stools. Therefore the quest for discovering an 'antidiarrheal drug' is still continuing. Results of clinical trials with racecadotril, probiotics and prebiotics are still inconclusive to support routine use of these agents in the treatment of diarrhea. A newer concept of calciumsensing receptor (CaSR) and its role in addressing all four major pathophysiological mechanisms of diarrheal disease is a way forward for future research [10]. Appropriate case management of diarrhea remains one of the essential components of Integrated Management of
Neonatal and Childhood Illness (IMNCI) strategy in Reproductive and Child Health Program of Govt. of India. Other approaches specifically focusing on Diarrhea and Pneumonia management as a priority are complementing the efforts made by the integrated approach.

\section{REFERENCES}

1. Udani PM, Shah PM, Mukerji S, Panvalkar RS, Kumbhat MM, Sanzgiri RR, et al. Trends in treatment of acute diarrhoeas in infancy. Indian Pediatr. 1968;5:1-16.

2. Portnoy BL, DuPont HL, Pruitt D, Abdo JA, Rodriguez JT. Antidiarrheal agents in the treatment of acute diarrhea in children. JAMA. 1976;236:844-6.

3. Jain TS, Mittal SK. Diarrhea and dehydration in pediatric practice. Indian Pediatr. 1977;14:401-4.

4. Bhargava SK, Sachdev HP, Das Gupta B, Daral TS, Singh HP, Mohan M. Oral rehydration of neonates and young infants with dehydrating diarrhea: comparison of low and standard sodium content in oral rehydration solutions. J Pediatr Gastroenterol Nutr. 1984;3:500-5.

5. Mittal SK. Oral rehydration: universal solution. Indian Pediatr. 1986;23:895-7.

6. Molla AM, Ahmed SM, Greenough WB 3rd. Rice-based oral rehydration solution decreases the stool volume in acute diarrhoea. Bull World Health Organ. 1985;63:751-6.

7. Brown KH, Gastanaduy AS, Saavedra JM. Effect of conti-nued oral feeding on clinical and nutritional outcome of acute diarrhea in children. J Pediatr. 1988;112:191-200.

8. World Health Organization. Clinical Management of Acute Diarrhea: WHO/UNICEF Joint Statement. WHO/FCH/ CAH/04.7, WHO, 2004.

9. Bhatnagar S, Bhandari N, Mouli UC, Bhan MK. Consensus statement of IAP National Task Force on Management of Acute Diarrhea: Status report. Indian Pediatr. 2004;41:335-48.

10. Harrell JE, Cheng SX. Inability to reduce morbidity of diarrhea by ORS: Can we design a better therapy? Pediatr Res. 2017;Nov 23:doi: 10.1038/pr.2017.295. 